\title{
Crater Density Predictions for New Horizons flyby target 2014 MU69
}

\author{
Sarah Greenstreet \\ B612 Asteroid Institute, 20 Sunnyside Ave, Suite 427, Mill Valley, CA 94941 \\ DIRAC Center, Department of Astronomy, University of Washington, 3910 15th Ave \\ NE, Seattle, WA 98195 \\ Brett Gladman \\ Department of Physics \& Astronomy, 6224 Agricultural Road, University of British \\ Columbia, Vancouver, British Columbia \\ William B. McKinnon \\ Department of Earth and Planetary Sciences and McDonnell Center for Space Sciences, \\ One Brookings Drive, Washington University, St. Louis, MO 63130, United States \\ J. J. Kavelaars \\ National Research Council of Canada, Victoria, BC, Canada \\ Kelsi N. Singer \\ Southwest Research Institute, 1050 Walnut Street, Suite 300, Boulder, CO 80302, United \\ States
}

\begin{abstract}
In preparation for the Jan 1/2019 encounter between the New Horizons spacecraft and the Kuiper Belt object 2014 MU69, we provide estimates of the expected impact crater surface density on the Kuiper Belt object. Using the observed crater fields on Charon and Pluto down to the resolution limit of the 2015 New Horizons flyby of those bodies and estimates of the orbital distribution of the crater-forming projectiles, we calculate the number of craters per unit area formed as a function of the time a surface on 2014 MU69 has been exposed to bombardment. We find that if the shallow cratersize distribution from 1-15 km exhibited on Pluto and Charon is indeed due to the sizes of Kuiper Belt projectiles, 2014 MU69 should exhibit a surface
\end{abstract}


that is only lightly cratered below $1 \mathrm{~km}$ scale, despite being bombarded for $\sim 4$ billion years. Its surface should therefore be more clearly indicative of its accretionary environment. In addition, this object may the first observed for which the majority of the bombardment is from exogenic projectiles moving less than or near the speed of sound in the target materials, implying morphologies more akin to secondary craters elsewhere in the Solar System. Lastly, if the shallow Kuiper Belt size distribution implied from the Pluto and Charon imaging is confirmed at MU69, then we conclude that this size distribution is a preserved relic of its state $\simeq 4.5$ Gyr ago and provides a direct constraint on the planetesimal formation process itself.

\section{Introduction}

The upcoming flyby of the New Horizons spacecraft by the cold classical transneptunian object (TNO) 2014 MU69 (hereafter referred to as MU69) will offer the first up-close look (sub-km resolution) of a small outer Solar System body in its formation environment, and will provide the first opportunity to observe a high-resolution cratered surface of a TNO other than those in the Pluto system, imaged by New Horizons in July 2015 (Stern et al., 2015; Moore et al., 2018).

The classical Kuiper belt appears to be divided into at least two separate inclination components (Brown, 2001). The 'cold' classical Kuiper belt (Kavelaars et al., 2008) contains the vast majority of the TNOs on loweccentricity orbits of low inclination in the semimajor axis range $42.4<a<$ 47 au (Petit et al., 2011). The existence of large separation TNO binaries combined with the low orbital inclination distribution suggests that the cold classicals represent a reservoir of the primordial proto-planetary disk beyond 40 au (Parker and Kavelaars, 2010). This the only sub-population thought to have been in place since the formation of the Solar System. The low orbital 
inclination and eccentricity of MU69's orbit (Porter et al., 2018) place this object firmly within this region and thus MU69 is likely to have formed at this large heliocentric distance and be the most primitive outer Solar System body yet visited. There is considerable structure in the $a$ and eccentricity (e) space of the cold population, and it is has been split (Petit et al., 2011) into a 'stirred' component crossing the whole cold $a$ range, and a much more confined 'kernel' of cold objects near $a=44$ au and $e \simeq 0.05$; MU69 is consistent with being in this kernel.

The other Kuiper Belt sub-populations (characterized by broader orbital inclination distributions) have been postulated to have formed closer to the Sun and transplanted to their current location in the final stages of planet formation (e.g. Levison et al., 2008). The postulated 500 Myr delay in the instability in the often-cited Nice Model (Gomes et al., 2005) has been retreated from (Mann, 2018); see Nesvorný (2018) for a recent review. The desirable implantation properties for creating Kuiper Belt structure, however, still hold even if the instability process occurs early in Solar System history (Gladman et al., 2012). In the scenario that MU69 has always been near its current orbit, a hypothetical metastable phase (which was of interest for Pluto's early cratering since Pluto is part of the metastable population) is irrelevant because during the early phase the population closer to the Sun is not cratering MU69. In either case, the 'hot' populations are ultimately emplaced during a relatively brief ( $<1 \%$ of the Solar System's age) period in which the cold population must not be dynamically excited (eg., Dawson and Murray-Clay, 2012). While we thus concentrate our calculations on the last 4 Gyr of bombardment, we argue below that the brief phase in which the objects where scattered out through the Kuiper belt, with some small fraction becoming the hot classical Kuiper belt we see today, cannot contribute a large change in MU69 cratering, especially given that we find 
that the cold populations dominate the impactor flux.

The observed crater density on Pluto and Charon by the New Horizons spacecraft during its flyby of the Pluto system in July 2015 showed a change to a shallower size distribution (Singer et al., 2019). We assume here that this transition is caused by a similar paucity of small impactors, and combine the derived impactor size distribution with a Kuiper belt orbit distribution model to compute the impactor flux, impactor speed distributions, and resulting crater formation rates on MU69.

\section{The impactor size distribution}

Calculating crater formation rates requires a knowledge of the impactor population's orbital distribution (which determines the impact probabilities and impact speed distribution) and its diameter $d$ distribution in order to compute the scale of the resulting craters of diameter $D$. The differential number of objects $d N$ as a function of absolute $H$-magnitude behaves locally as $d N \propto 10^{\alpha H}$, where the exponential index $\alpha$ is also referred to as the logarithmic "slope" (hereafter referred to simply as the slope). This corresponds to a cumulative power law distribution in the projectile diameter with $N(<d) \propto d^{-5 \alpha}$. The conversion for objects with absolute g-band magnitude $H_{g}$ and scaling to an albedo $p$ of $5 \%$ is

$$
d \simeq 100 \mathrm{~km} \sqrt{\frac{0.05}{p}} 10^{0.2\left(9.16-H_{g}\right)}
$$

where we see that $H_{g}=9.16$ corresponds to $100 \mathrm{~km}$. We have chosen to make a single pre-encounter crater-density prediction, based on telescopic observations and the results of the New Horizons flyby of Pluto and Charon. We use one size distribution (Fig. 1) for all of the various Kuiper Belt subpopulations. The steep observed Kuiper Belt size distribution for TNOs $d>100 \mathrm{~km}$ rolls at this diameter to a "knee" size distribution, as used 


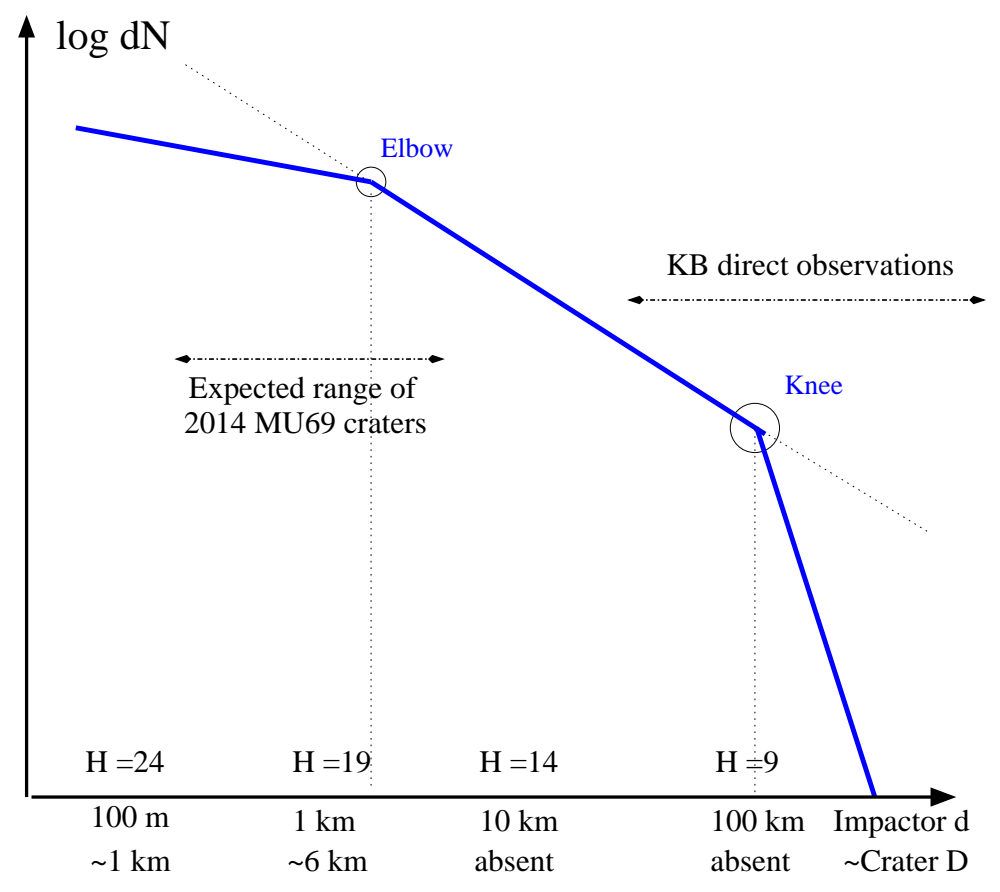

Figure 1: Cartoon schematic of the $H_{g}$-magnitude differential size distribution. The Kuiper belt observations are well calibrated down to $H_{g} \approx 10$, but smaller than the knee at $H_{g} \sim 9$ (open circle) the size distribution flattens from a slope of $\alpha \simeq 0.8$ (not relevant to this paper) down to a shallower $\alpha \simeq 0.4$. Singer et al. (2019) find the impactor size distribution from craters observed on Charon requires a second change to a slope of $\alpha \simeq 0.15$ for $d \lesssim 2 \mathrm{~km}$. For reference, impactor diameters $d$ are converted to rough MU69 crater diameters $D$ assuming a common impact speed of $300 \mathrm{~m} / \mathrm{s}$. The expected range of craters observable by New Horizons covers $D \approx 200 \mathrm{~m}$ to $D \approx 20 \mathrm{~km}$ (created by cold-belt impactors ranging from $d \approx 0.02-3 \mathrm{~km}$ ). Notice that the ratio of crater to impactor diameters is smaller than usual due to the low impact speeds.

in Greenstreet et al. (2015, 2016); the slope $\alpha=0.4$ below this knee extends down to another slope change at a scale which Singer et al. (2019) denote as the "elbow", visible on both Pluto and Charon. On Charon, this corresponds to a crater size of $D \approx 13 \mathrm{~km}$, which for typical Charon impact speeds implies projectile $d \approx 2 \mathrm{~km}$ and thus $H_{g} \approx 17.5$. We connect the $H_{g}<9.16$ population estimates from Petit et al. (2011) and Gladman et al. (2012) to the $H_{g}<17.5$ scale by using a multiplicative factor of $10^{\alpha \Delta H}=$ $10^{((0.4 * 8.5)-(0.8 * 0.16))} \approx 1,900$. Below this scale Pluto and Charon show a very 
shallow distribution with $\alpha \simeq 0.15$, and we assume this slope is that coming from the impactor size distribution and directly map it back to the projectiles. Fig. 1s horizontal axis also shows the expected crater diameter caused by a typical cold-population impactor striking MU69 (see below). If this crater size distribution is in fact present on MU69 it will be a dramatic confirmation of a shallow Kuiper Belt size distribution in the roughly $0.1-2 \mathrm{~km}$ diameter range.

\section{Methods}

The methods for computing the current impact fluxes and cratering rates onto MU69 are identical to those in Greenstreet et al. (2015, 2016), to which we refer the reader for the details; below we only mention any deviations from the methods used in the previous paper.

\subsection{Kuiper belt population models}

MU69 sits in the heart of the cold classical Kuiper Belt's kernel with the encounter target having $a=44.2 \mathrm{AU}, e=0.04$, and heliocentric J2000 orbital inclination $i=2.4^{\circ}$ (Porter et al., 2018). We adopted the same orbital distributions and $N\left(H_{g}<9.0\right)$ population estimates for the various Kuiper Belt sub-populations as before and computed the impact fluxes onto MU69. We added to the analysis the population of TNOs in the 7:4 meanmotion resonance with Neptune (whose population is small and was thus not used for the Pluto/Charon analysis), because the resonant semimajor axis of $a \simeq 43.6 \mathrm{AU}$ is close to MU69 and the impact probability per particle is enhanced, thus contributing a non-negligible fraction of the total impact flux onto MU69.

As in Greenstreet et al. (2015, 2016), we account for the slow decay of each projectile sub-population over the last 4 Gyr when we compute the total impact flux. For the 7:4 resonance we adopt the same decay as for the 2:1 
objects found in Tiscareno and Malhotra (2009). These enhancements back in time are modest except for the scattering TNO population, but we show below that this population is only a tiny contributor to MU69 cratering.

\section{2. Öpik collision probability code}

The Öpik collision probability code used in this study is the same as used previously, with the only change being the new target body. MU69's gravitational focusing is negligible, but is included. Unlike computing the impact flux onto Pluto from the various sub-populations, 2014 MU69 is not located within a mean-motion resonance nor is it undergoing Kozai oscillations. It was thus not necessary to provide a correction factor to the collision probabilities provided by the Öpik collision probability code to take into account how the dynamics allowed Pluto to avoid orbital intersections with some portions of some sub-populations. Our code provides the impact probabilities for the ensemble of objects in each orbit and the probability-weighted impact speed distribution onto MU69.

The impact speed distribution is remarkable (Fig. 2). Essentially all impacts onto MU69 are less than the $\sim 4 \mathrm{~km} / \mathrm{s} p$ wave speed in solid water ice; thus if MU69 is a coherent body these impacts are subsonic, where the crater scaling laws are less well-established (see discussion below and in Singer et al. (2013)). The most extreme behavior is that, because of their similar orbits to MU69, the cold classicals have tiny impact speeds, peaking around only $300 \mathrm{~m} / \mathrm{s}$, similar to the $p$ wave speed in unconsolidated sands - which may be a better structural analog to MU69's surface. While such speeds are above MU69's escape speed, they are essentially unknown for primary projectiles in the Solar System and would only be seen elsewhere in the context of non-escaping, secondary projectiles. Fig. 2 shows, as expected, the 7:4 resonant objects have a range of encounter speeds including serendipitous close encounters with MU69 at similarly low velocities due to their very sim- 


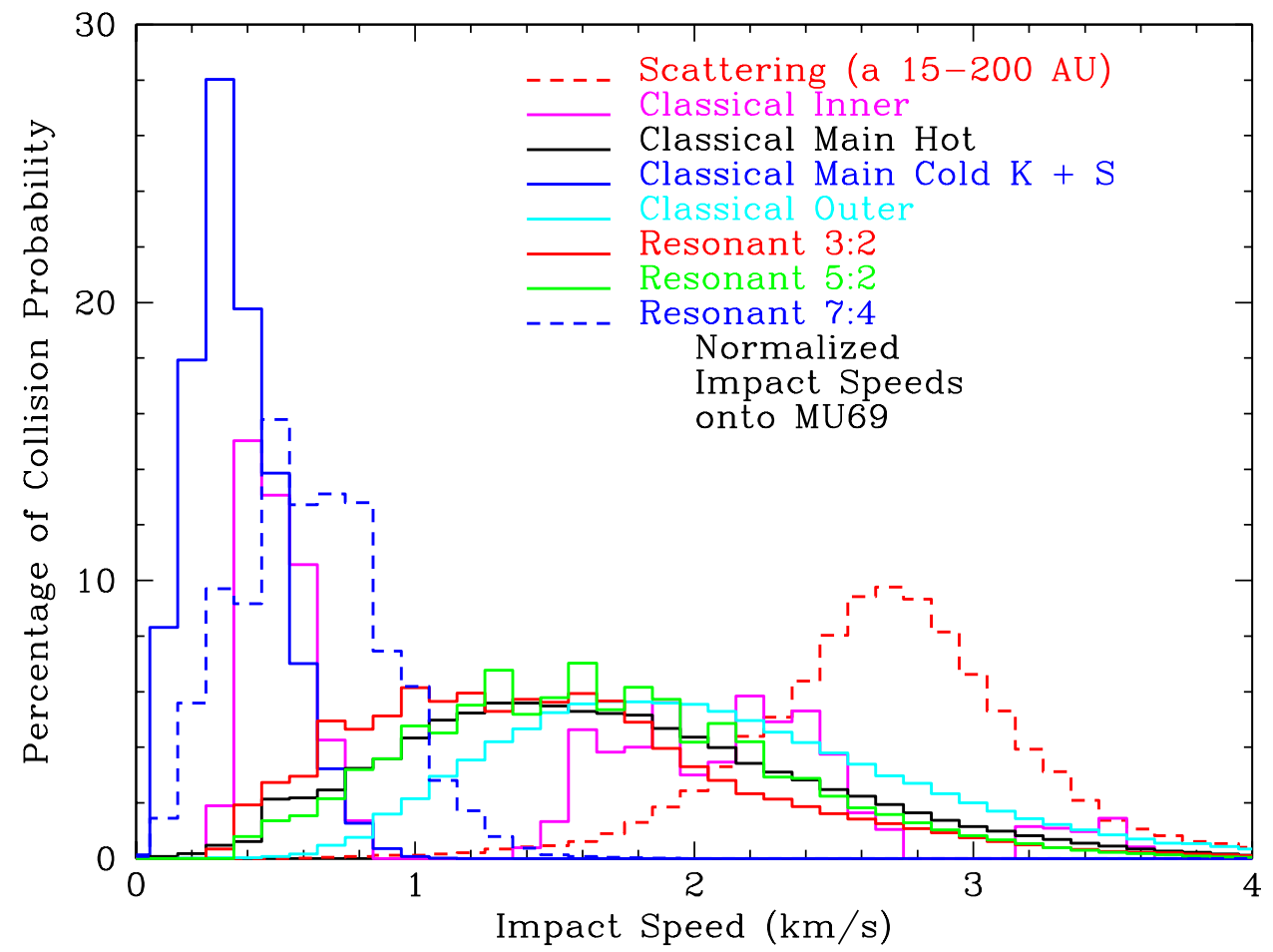

Figure 2: Impact velocity spectrum onto MU69 for each Kuiper Belt sub-population. Each sub-population's distribution is separately normalized. The cold main classical impact velocity distribution includes both the stirred and kernel sub-components, because their speed distributions are similar. The bimodal nature of the (nearly negligible) inner classical belt is due to a gap in that population's orbital inclination distribution (Petit et al., 2011). Almost all impactors on MU69 are travelling less than the speed of sound in coherent water ice.

ilar semimajor axes. Unsurprisingly the scattering objects, which have large semimajor axes, peak at the highest impact velocity $(\approx 3 \mathrm{~km} / \mathrm{s})$ of the subpopulations. The remaining sub-populations peak at similar impact speeds near $\approx 1.5 \mathrm{~km} / \mathrm{s}$.

\subsection{Impact rates onto MU69}

Table 1 shows the calculated Öpik collision probabilities (/yr/TNO) onto MU69 for each Kuiper Belt sub-population (this collision probability is independent of the projectile size). This is converted to an impact rate by multiplying by the estimated corresponding population at some scale. To 
pick a single number, we have chosen to tabulate the rate of projectiles of the size scale of the elbow or larger $\left(H_{g}<17.5\right.$ or $\left.d \gtrsim 2 \mathrm{~km}\right)$. This reveals that the impact rate is dominated by the three components of the main classical belt, which together supply $(10+58+18)=86 \%$ of the projectiles that have struck MU69 over the last 4 Gyr. The total impact rate of projectiles larger than the elbow is (perhaps surprisingly) low at only $0.017 / \mathrm{Gyr}$; it is thus likely that over the entire post-accretionary bombardment history no projectile with $d \gtrsim 1 \mathrm{~km}$ has struck MU69 (we assume an effective target radius of $10 \mathrm{~km}$ ). We thus predict that large craters will be rare or absent; we quantify this below. The impact flux from impactors smaller than the elbow will of course be larger due to their increasing numbers as diameters drop, but with a shallow slope they do not become numerous quickly. Our calculations of crater production that follow peform the full integration over the size and speed distributions, and are thus better than quoting a single number.

Another remarkable result shown in Table 1 is that the scattering population is a fractionally negligible addition to the impact rate. The resonant populations are each small contributors. The large 3:2 population (which was the most important of the sub-populations for Pluto cratering) has a low impact probability per particle due to the large mutual orbital inclination with MU69. The enhancement of the 7:4 population (by about a factor of two per particle) is evident, but the four most important resonant sources together provide $<10 \%$ of the impactor flux. The classical inner population is essentially negligible because many of its members do not intersect the MU69 orbit. Finally, the population of non-resonant TNOs with semimajor axes beyond the 2:1 resonance (the 'Classical Outer' population) contributes about $6 \%$ of the impactors; this population has many orbits with perihelion just inside MU69's perihelion and as Fig. 2 shows these impactors have 
median impact speeds $U \sim 2 \mathrm{~km} / \mathrm{s}$.

\begin{tabular}{|c|c||c|c|c||c|c|}
\hline $\begin{array}{c}\text { Kuiper Belt } \\
\text { Sub-Population } \\
\text { Type }\end{array}$ & $\begin{array}{c}H_{g}<17.5 \\
\text { Population } \\
\text { Estimate }\end{array}$ & $\begin{array}{c}\text { Öpik } \\
\text { Impact } \\
\text { Probability } \\
(/ \mathbf{y r} / \mathbf{T N O})\end{array}$ & $\begin{array}{c}d \gtrsim 2 \mathbf{k m} \\
\text { Impact } \\
\text { Rate } \\
(/ \mathbf{G y r})\end{array}$ & $\begin{array}{c}\text { \% of } \\
\text { Total } \\
\text { Impact } \\
\text { Rate }\end{array}$ & $\begin{array}{c}D>1 \mathbf{k m} \\
\text { Cratering } \\
\text { Rate } \\
(/ \mathrm{Gyr})\end{array}$ & $\begin{array}{c}\text { \% of } \\
\text { Total } \\
\text { Cratering } \\
\text { Rate }\end{array}$ \\
\hline $\begin{array}{c}\text { S.O. (15 AU } \leq \\
a \leq 200 \mathrm{AU})\end{array}$ & $2.0 \mathrm{e} 7$ & $3.4 \mathrm{e}-21$ & $6.8 \mathrm{e}-5$ & 0.4 & $7.3 \mathrm{e}-3$ & 0.7 \\
\hline S.O. $(a>200 \mathrm{AU})$ & $1.8 \mathrm{e} 8$ & $1.0 \mathrm{e}-22$ & $1.8 \mathrm{e}-5$ & 0.1 & $2.1 \mathrm{e}-3$ & 0.2 \\
\hline Classical Inner & $5.5 \mathrm{e} 6$ & $5.6 \mathrm{e}-22$ & $3.1 \mathrm{e}-6$ & 0.0002 & $2.6 \mathrm{e}-4$ & 0.0002 \\
\hline Classical Main H & $6.5 \mathrm{e} 7$ & $2.4 \mathrm{e}-20$ & $1.6 \mathrm{e}-3$ & $\mathbf{9 . 5}$ & $1.4 \mathrm{e}-1$ & $\mathbf{1 3 . 2}$ \\
\hline Classical Main S & $1.4 \mathrm{e} 8$ & $7.0 \mathrm{e}-20$ & $9.8 \mathrm{e}-3$ & $\mathbf{5 8 . 0}$ & $5.4 \mathrm{e}-1$ & $\mathbf{5 0 . 7}$ \\
\hline Classical Main K & $3.8 \mathrm{e} 7$ & $7.8 \mathrm{e}-20$ & $3.0 \mathrm{e}-3$ & $\mathbf{1 7 . 8}$ & $1.6 \mathrm{e}-1$ & $\mathbf{1 5 . 0}$ \\
\hline Classical Outer & $1.5 \mathrm{e} 8$ & $6.8 \mathrm{e}-21$ & $1.0 \mathrm{e}-3$ & 5.9 & $9.9 \mathrm{e}-2$ & 9.3 \\
\hline Resonant 3:2 & $2.5 \mathrm{e} 7$ & $2.7 \mathrm{e}-20$ & $6.8 \mathrm{e}-4$ & 4.0 & $5.8 \mathrm{e}-2$ & 5.4 \\
\hline Resonant $2: 1$ & $7.0 \mathrm{e} 6$ & $2.3 \mathrm{e}-20$ & $1.6 \mathrm{e}-4$ & 0.9 & $1.4 \mathrm{e}-2$ & 1.4 \\
\hline Resonant $5: 2$ & $2.3 \mathrm{e} 7$ & $1.2 \mathrm{e}-20$ & $2.8 \mathrm{e}-4$ & 1.7 & $2.6 \mathrm{e}-2$ & 2.4 \\
\hline Resonant 7:4 & $5.5 \mathrm{e} 6$ & $5.0 \mathrm{e}-20$ & $2.8 \mathrm{e}-4$ & 1.7 & $1.8 \mathrm{e}-2$ & 1.7 \\
\hline Total & & & $\mathbf{0 . 0 1 7}$ & $\mathbf{1 0 0 . 0}$ & $\mathbf{1 . 1}$ & $\mathbf{1 0 0 . 0}$ \\
\hline
\end{tabular}

Table 1: Öpik collision probability calculations, $d \gtrsim 2 \mathrm{~km}$ impact rates, and $D>1 \mathrm{~km}$ cratering rates onto MU69 for each Kuiper Belt sub-population. Population estimates are for $H_{g}<17.5$ (diameter $d \gtrsim 2 \mathrm{~km}$, for a g-band albedo $p=5 \%$ ), which is the location of the elbow. Impact probabilities are (/yr/object). Impact rates are (/Gyr) and determined using the number of $H_{g}<17.5$ objects in each sub-population assuming an effective target radius near $10 \mathrm{~km}$ (the stellar occultation silhouette is $20 \mathrm{x} 35 \mathrm{~km}$, but irregular (Moore et al., 2018)). Cratering rates for $D>1 \mathrm{~km}$ are (/Gyr) of bombardment.

Our derived impact fluxes, when expressed as impacts per year per $\mathrm{km}^{2}$ of target (assuming an effective spherical radius of $r \approx 10 \mathrm{~km}$ ), are about twice that onto Pluto, reflecting the higher spatial density of the MU69 environment. This relative flux is in agreement with JeongAhn et al. (2018, to be submitted) who also conclude that the cold classicals should provide $\sim 80 \%$ of the impacts.

\subsection{Cratering rates onto MU69}

For the purposes of this paper we assume that MU69 is an unconsolidated, low-density body. We convert between crater diameters $D$ and projectile 
diameter $d$ via

$$
D=8.9\left(\frac{U_{k m / s}^{2}}{g_{c m / s^{2}}}\right)^{0.170}\left(\frac{\delta}{\rho}\right)^{0.333} d_{k m}^{0.830} \mathrm{~km}
$$

where $U$ is the impact velocity (in $\mathrm{km} / \mathrm{s}$ ), $d$ is the impactor diameter, and the gravitational acceleration on the surface of MU69 is $g \approx 0.3 \mathrm{~cm} / \mathrm{s}^{2}$. We assume both impactor $\delta$ and target $\rho$ (at surface) densities are $\delta=\rho=$ $1.0 \mathrm{~g} / \mathrm{cm}^{3}$, although the value is unimportant as long as they are the similar.

The coefficients in Eq. (2) are appropriate to unconsolidated sand or the regolith in the gravity regime (Singer et al., 2013), and are based on wellestablished scaling from laboratory and numerical simulations (Holsapple, 1993; Housen and Holsapple, 2011; Greenstreet et al., 2015, 2016; Singer et al., 2019). This procedure should provide a reasonably accurate estimate of crater size (to within $+/-50 \%$ ), provided neither the impact speed nor the cratering efficiency (excavated crater mass/impactor mass) are too low. Well-defined secondary craters are seen on icy satellites for speeds down to $\approx 200 \mathrm{~m} / \mathrm{s}$, so we anticipate that most TNO impacts onto MU69 will form craters; the lowest-speed impactors may be accretionary, however, not crater-forming. Despite the difference of physical regime, Eq. 2 gives quite similar results to those obtained if we use the scaling law we deployed in Greenstreet et al. (2015) appropriate for non-porous coherent surfaces; when propagated through the entire analysis the resulting crater densities presented below are nearly identical. Thus, uncertainties in the scaling law is of little consequence compared to the much larger uncertainties that we expect to arise because of the sparse crater statistics.

First note that for the tiny $U \simeq 0.3 \mathrm{~km} / \mathrm{s}$, craters are only $D \simeq 7 \mathrm{~km}$ for a $d=1 \mathrm{~km}$ projectile, and thus the crater/projectile ratio is somewhat smaller than the typical 10-20 common elsewhere in the solar system. By integrating the crater production over all speeds of all sub-populations, we 


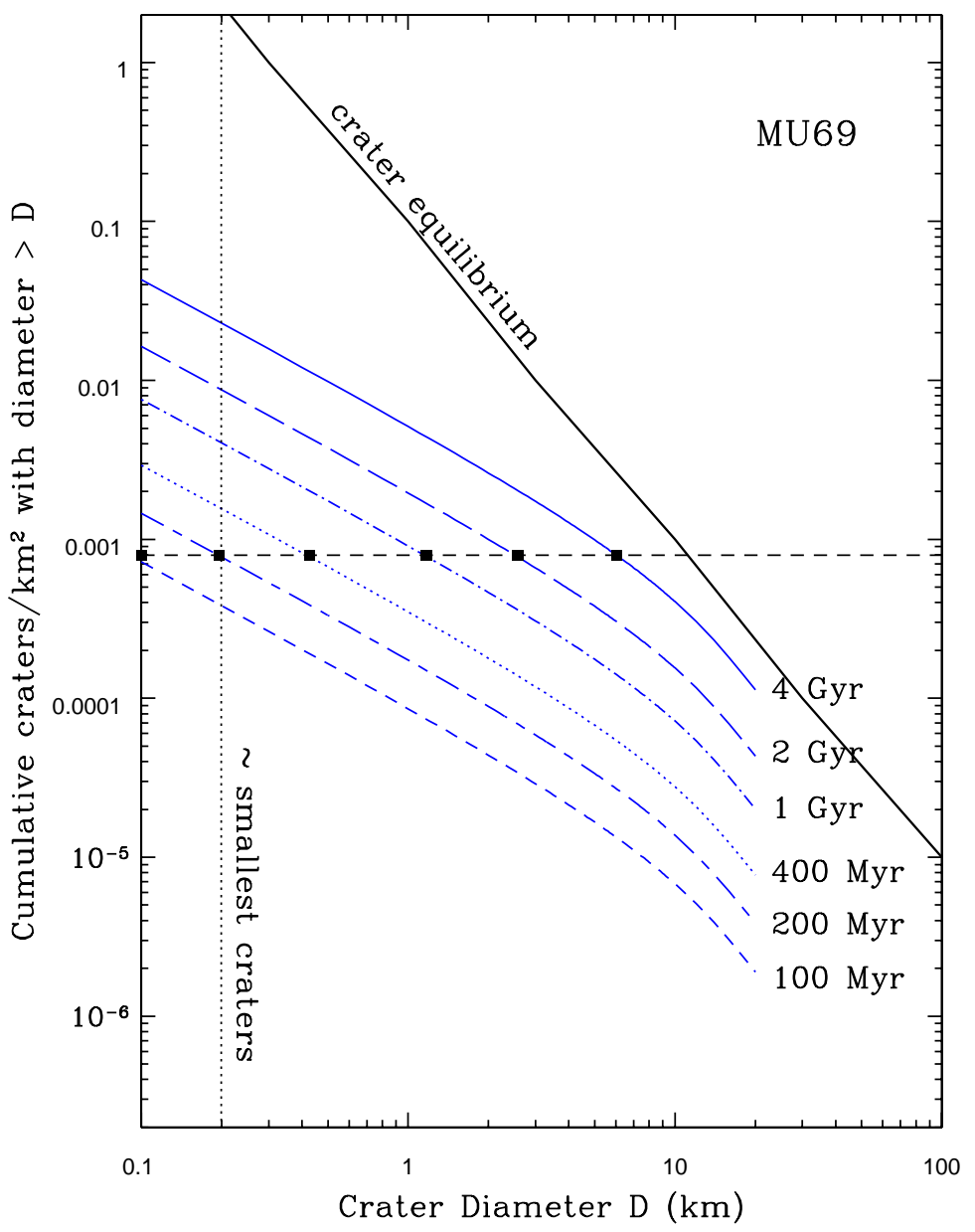

Figure 3: Logarithm of crater density $\left(\#\right.$ craters $\left./ \mathrm{km}^{2}\right)$ larger than a given crater diameter $D$ on MU69's surface versus the logarithm of the crater diameter for an impactor size distribution with a both a knee (not visible in this plot) and elbow (shown as the gradual slope change near $D \approx 10 \mathrm{~km}$ ) at various surface ages. The solid black line is the crater equilibrium curve from Melosh (1989). The horizontal line at $\approx 10^{-3}$ corresponds to 1 crater/MU69 surface, and the vertical line is an estimate for the smallest craters that will be visible in the best MU69 images. The black squares at the 1 crater/MU69 surface line correspond to the black squares in Figure 4. The change in slope at $D \approx 10 \mathrm{~km}$ corresponds to the elbow break in the impactor size distribution, but will not be visible in the crater data; note that this change is gradual because of the large fractional spread in impact speeds $U$ present. 
derive the crater production rates shown in Fig. [3 for various bombardment time scales.

Although a single number is of limited utility, Table 1 also lists the rate of production of impact craters larger than an arbitrarily-chosen size of $D>$ $1 \mathrm{~km}$ from each projectile sub-population. This crater formation rate is low. Accounting for all the projectiles, the expectation is a modest 5 craters with $D>1 \mathrm{~km}$ on MU69 after bombardment for the Solar System's age. Because of the low impact speeds from the cold classical sources, their dominance is somewhat diminished in terms of fraction of the crater production above a given $D$ limit, as their craters are smaller than those produced by the higher-speed populations; the cold population still manages to contribute three-quarters of the $D>1 \mathrm{~km}$ production.

Fig. 3 provides a much more complete view of our results in the form of predicted cumulative crater density curves, for several bombardment durations (that is, ages since a surface was last reset by geologic processes). The horizontal line corresponds to 1 crater on the surface. The conclusion is dramatic: MU69 should be lightly cratered near the resolution limit, for despite extremely low gravity on MU69, the paucity of small projectiles and their slow impact speeds produce few craters with $D \sim 200 \mathrm{~m}$. Even more spectacularly, given that the surface area of MU69 is only $\sim 1000-2000 \mathrm{~km}^{2}$, the 4 Gyr bombardment predicts only $\sim 25-50$ craters to be above the effective $\sim 200 \mathrm{~m}$ resolution limit of MU69, and note that only half of the body is likely to get this highest-resolution data. As long as craters are visible, however, this predicted level of cratering would provide strong confirmation that the paucity of Pluto/Charon craters smaller than the elbow scale (at crater $D<13 \mathrm{~km}$ for Pluto/Charon or impactor $d \lesssim 2 \mathrm{~km}$ ) is indeed a feature of the projectile size distribution. At the large end, bombardment over the entire age of the Solar System will on average provide a crater with 
a maximum diameter of order $6 \mathrm{~km}$ (a larger crater could be possible due to Poisson statistics).

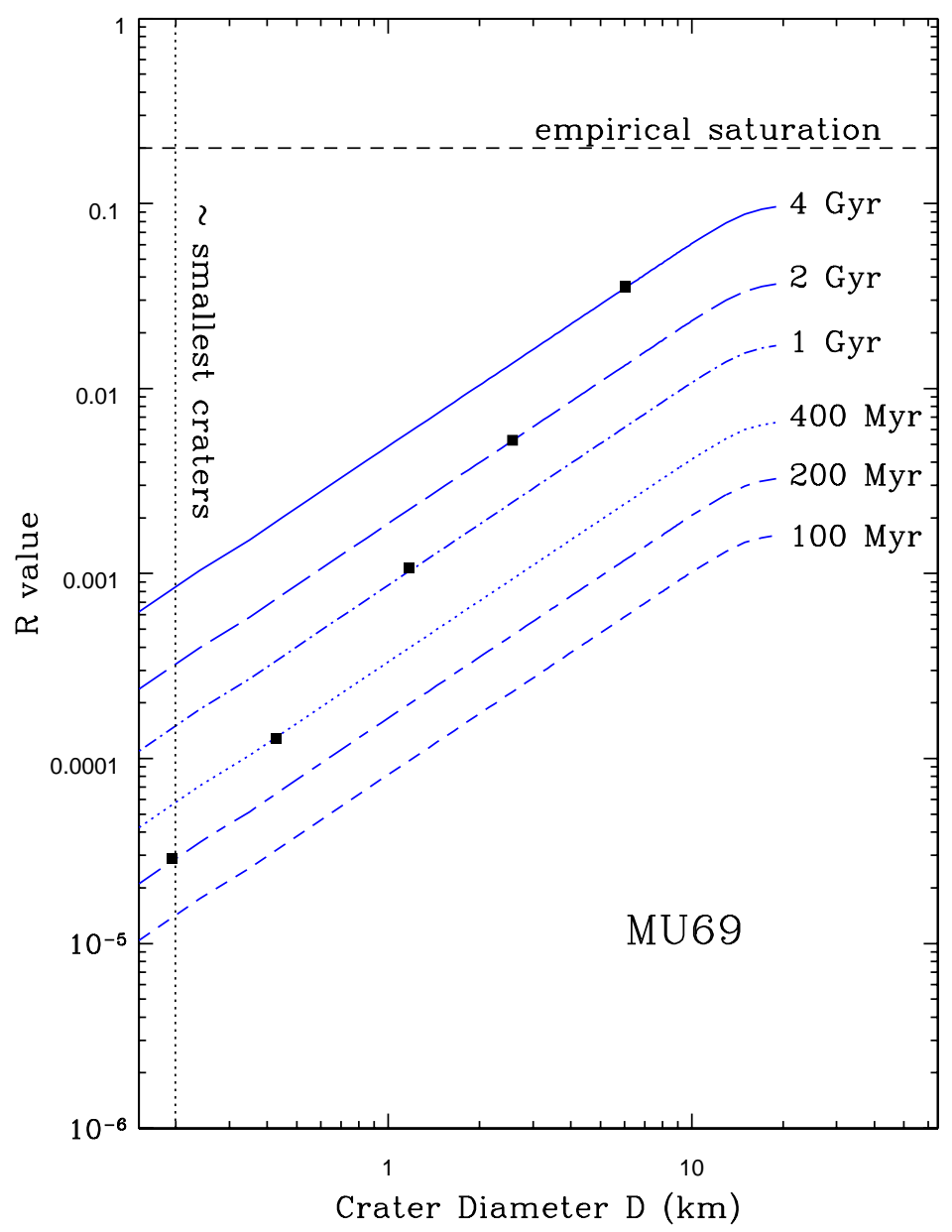

Figure 4: Relative crater frequency plot of the same information in Figure 3 , The black squares correspond to 1 crater/MU69 surface on the cumulative plot (Figure 3), so craters larger than the dots will likely not be visible on MU69's post-accretionary terrains, except by statistical fluctuation. The fact that $\mathrm{R}$ values rise for increasing $D$ is due to the relatively shallow projectile size distribution implied by the Pluto/Charon surfaces. Assuming MU69 preserves the craters integrated over all of Solar System history, this level of cratering in the visible portion will be sparse statistically.

Fig. 4 displays our results in 'R-plot' formulation, which can be thought of as roughly the fraction of the surface covered in craters at each crater scale 
$D$. The rise with increasing diameter over the visible range would be very characteristic of the projectile population derived from THE Pluto/Charon crater data. If the (probably sparse) crater counts do indeed match these predicted values, it would strongly argue that the current Kuiper belt size distribution is extremely shallow in the $100 \mathrm{~m}$ to $1 \mathrm{~km}$ diameter range probed by MU69 and the Pluto-Charon crater counts. This would then offer the strong possibility that the current Kuiper Belt retains the primordial planetesimalbuilding size distribution.

\section{Discussion and Conclusion}

Based on the above results, we conclude that bombardment over the entire age of the Solar System is insufficient to more than modestly crater 2014 MU69 near the expected resolution limit. That is, if during its formation process, accretion activity was sufficient to erase any craters that may have been acquired during its assembly, MU69 will be modestly cratered today. It would thus be incorrect to conclude that a lightly cratered surface implies that MU69's surface has been recently reset (or more extremely, that MU69 has been recently created as a collisional fragment from a larger TNO). Charon's extensive cryovolcanic plain (informally named Vulcan Planitia) is an example of this; it is unsaturated and the observed crater densities correspond to those expected from bombardment for $\approx 4$ billion years (Singer et al., 2019).

Because of this light cratering, MU69 exists in an environment very different from main-belt asteroids of comparable $(\sim 10 \mathrm{~km})$ size. The latter are thought to essentially all be fragments produced after the accretionary epoch, and thus are not 'primordial' objects directly. In contrast, MU69 will now look very much like it did at its formation epoch (in the sense that impact processes have not made global structural changes to the body nor even greatly affected more than a modest fraction of the surface). Despite MU69's 
low gravity, even the largest craters that form are unlikely to provide enough distant ejecta to erase 200 m craters; ejecta dispersal will be even more limited if MU69 is very porous, as seen at asteroid Mathilde (Veverka et al., 1999).

Looking beyond the values of the crater densities themselves, no solar system body has ever been studied where the majority of the primary cratering is from significantly subsonic projectiles. This could result in craters with lowered depth/diameter ratios as seen for secondary craters (e.g., Bierhaus and Schenk, 2010), and perhaps produce a morphologically visible difference between craters formed by impactors coming in from the cold-classical population versus those which tend to be in the $3-4 \mathrm{~km} / \mathrm{s}$ range. Seeing such differences will require well-resolved craters, which we expect to be modest in number even in the best MU69 images. For the slowest impact speeds, we do not expect crater formation at all, but rather, accretion of the impactor material, either as coherent mounds or dispersed debris fields ("paintball" patterns). In principle these speed differences might be able to help distinguish between craters (or other features) formed by cold classical (kernel or stirred) impactors versus those from the other sub-populations.

The uncertainty in the details of the dynamical state of the early outer Solar System is only a small concern to our interpretation, due to the dominance of the cold component's impactors. Under a scenario that all the hot populations were 100 times more populous for a brief ( $~ 50$ Myr) of Solar System history (and a $100 \times$ enhancement throughout that entire interval is unlikely), this would still only double hot-population contributions to the cratering rate; Table 1 shows that even in this case the cold classical projectiles still dominate and the total cratering only rises by a few tens of percent. This is insufficient to qualitatively alter our conclusions that MU69 will be dominated by subsonic projectiles and modestly cratered. If the object is 
heavily cratered, our interpretation would be that this is a crater field preserved from the initial assembly epoch of MU69 itself during the phase in which it accreted from smaller components.

Lastly, and most exciting, if the crater density and diameter distribution are consistent with bombardment over the age of the Solar System by the projectile distribution we derive based on the Pluto and Charon cratering results, this will serve as convincing proof of a very shallow sub-km size distribution in the Kuiper Belt itself (or, at least, in the cold population which dominates the MU69 crater production rate). Although Singer et al. (2019) already argue against the idea that surface geology on Pluto and Charon could create the shallow size distribution near and below the elbow scale (a crater size distribution which is similar across a variety of terrains on both bodies), such crater degradation ideas would be extremely unlikely to work in the same way on tiny MU69. A signature with crater $R$ values $\sim 0.001-0.01$ that are increasing as one moves to larger crater diameters would confirm that the elbow and its slope as seen on Pluto/Charon are due to a transition to a shallow projectile size distribution. If so, then not only is MU69 and its surface in a largely primordial state, but the size distribution of the projectiles themselves is also a largely un-evolved relic of the formation epoch. This unusual state is possible because the $\alpha \simeq 0.15$ distribution is so shallow there are not enough projectiles to disrupt the rapidlly shrinking targets as one moves down the size distribution. The current Kuiper Belt size distribution (at least of the cold population) would then be that which planetesimal accretion models would have to create, and the cratering record preserved on the already-imaged objects is directly providing constraints on the planet-building process. 


\section{Acknowledgements}

S. Greenstreet acknowledges support from the B612 Foundation. B. Gladman acknowledges support from the Canadian Natural Sciences and Engineering Research Council. W.B. McKinnon, J.J. Kavelaars, and K.S. Singer acknowledge support from the New Horizons project.

\section{References}

Bierhaus, E. B., Schenk, P. M., 2010. Constraints on Europa's surface properties from primary and secondary crater morphology. Journal of Geophysical Research 115, 12004.

Brown, M. E., May 2001. The Inclination Distribution of the Kuiper Belt. The Astronomical Journal 121, 2804-2814.

Dawson, R. I., Murray-Clay, R., May 2012. Neptune's Wild Days: Constraints from the Eccentricity Distribution of the Classical Kuiper Belt. The Astrophysical Journal 750, 43.

Gladman, B., Lawler, S. M., Petit, J. M., Kavelaars, J., Jones, R. L., Parker, J. W., Van Laerhoven, C., Nicholson, P., Rousselot, P., Bieryla, A., Ashby, M. L. N., 2012. The Resonant Trans-Neptunian Populations. The Astronomical Journal 144, 23-47.

Gomes, R., Levison, H. F., Tsiganis, K., Morbidelli, A., 2005. Origin of the cataclysmic Late Heavy Bombardment period of the terrestrial planets. Nature 435, 466-469.

Greenstreet, S., Gladman, B., McKinnon, W. B., Sep. 2015. Impact and cratering rates onto Pluto. Icarus 258, 267-288. 
Greenstreet, S., Gladman, B., McKinnon, W. B., Aug. 2016. Corrigendum to "Impact and Cratering Rates onto Pluto" [Icarus 258 (2015) 267-288]. Icarus $274,366-367$.

Holsapple, K. A., 1993. The scaling of impact processes in planetary sciences. Annual review of earth and planetary sciences 21, 333-373.

Housen, K. R., Holsapple, K. A., 2011. Ejecta from impact craters. Icarus $211,856-875$.

Kavelaars, J., Jones, L., Gladman, B., Parker, J. W., Petit, J.-M., 2008. The Orbital and Spatial Distribution of the Kuiper Belt. In: Barucci, M. A., Boehnhardt, H., Cruikshank, D. P., Morbidelli, A., Dotson, R. (Eds.), The Solar System Beyond Neptune. University of Arizona Press, pp. 59-69.

Levison, H. F., Morbidelli, A., Van Laerhoven, C., Gomes, R., Tsiganis, K., 2008. Origin of the structure of the Kuiper belt during a dynamical instability in the orbits of Uranus and Neptune. Icarus 196, 258-273.

Mann, A., Jan. 2018. Bashing holes in the tale of Earth's troubled youth. Nature 553, 393-395.

Melosh, H. J., 1989. Impact cratering: A geologic process. Oxford University Press, New York.

Moore, J. M., McKinnon, W. B., Cruikshank, D. P., et al., 2018. Great Expectations: Plans and Predictions for New Horizons Encounter With Kuiper Belt Object 2014 MU69 ("Ultima Thule"). Geophysical Research Letters 45, 8111-8120.

Nesvorný, D., Sep. 2018. Dynamical Evolution of the Early Solar System. Annual Review of Astronomy and Astrophysics 56, 137-174. 
Parker, A. H., Kavelaars, J. J., Oct. 2010. Destruction of Binary Minor Planets During Neptune Scattering. The Astrophysical Journal Letters 722, L204-L208.

Petit, J. M., Kavelaars, J. J., Gladman, B. J., Jones, R. L., Parker, J. W., Van Laerhoven, C., Nicholson, P., Mars, G., Rousselot, P., Mousis, O., Marsden, B., Bieryla, A., Taylor, M., Ashby, M. L. N., Benavidez, P., Campo Bagatin, A., Bernabeu, G., 2011. The Canada-France Ecliptic Plane Survey - Full Data Release: The Orbital Structure of the Kuiper Belt. The Astronomical Journal 142, 131-155.

Porter, S. B., Buie, M. W., Parker, A. H., Spencer, J. R., Benecchi, S., Tanga, P., Verbiscer, A., Kavelaars, J. J., Gwyn, S. D. J., Young, E. F., Weaver, H. A., Olkin, C. B., Parker, J. W., Stern, S. A., 2018. Highprecision Orbit Fitting and Uncertainty Analysis of (486958) 2014 MU69. The Astronomical Journal 156, 20-26.

Singer, K. N., McKinnon, W. B., Gladman, B., et al., 2019. Impact Craters on Pluto and Charon Reveal a Deficit of Small Kuiper Belt Objects. Science.

Singer, K. N., McKinnon, W. B., Nowicki, L. T., 2013. Secondary craters from large impacts on Europa and Ganymede: Ejecta size-velocity distributions on icy worlds, and the scaling of ejected blocks. Icarus 226, 865-884.

Stern, S. A., Bagenal, F., Ennico, K., et al., 2015. The Pluto system: Initial results from its exploration by New Horizons. Science 350, 1815.

Tiscareno, M., Malhotra, R., 2009. Chaotic Diffusion of Resonant Kuiper Belt Objects. The Astronomical Journal 138, 827-837. 
Veverka, J., Thomas, P., Harch, A., Clark, B., Bell, J. F., Carcich, B., Joseph, J., Murchie, S., Izenberg, N., Chapman, C., Merline, W., Malin, M., McFadden, L., Robinson, M., 1999. NEAR Encounter with Asteroid 253 Mathilde: Overview. Icarus 140, 3-16. 